\title{
COMPARING INTERFERON-GAMMA RELEASE ASSAY WITH TUBERCULIN SKIN TEST FOR IDENTIFYING LATENT TUBERCULOSIS INFECTION BETWEEN CHILDREN $<5$ YEARS AND $\geq 5$ YEARS OLD
}

Jong-Hyun Kim¹ , Ji Hyen Hwang'1, Ki Hwan Kim¹ , Byung-Wook Eun² , Hye-Kyung Cho3, Yun-Kyung Kim4, Su Eun Park5

Department of Pediatrics, 'The Catholic University of Korea, Seoul; ${ }^{2}$ Eulji University Nowon Hospital, Seoul; 3 Gachon University, Graduate School of Medicine, Incheon; 4Korea University Ansan Hospital, Ansan; 5Pusan National University Children's Hospital, Pusan National University School of Medicine, Yangsan; the Republic of Korea

\section{BACKGROUNDS}

The use of the interferon-gamma release assay (IGRA) for the diagnosis of latent tuberculosis infection in children under 5 years old is limited due to the low sensitivity.

$\checkmark$ We compared the results of tuberculin skin test (TST) and IGRA in children under 5 years and over 5 years old who had vaccinated BCG within 4 weeks of birth in a Korean national immunization program.

\section{METHODS}

From December 2016 to May 2017, we included the patients under 19 years old who were agreed to perform an IGRA in addition to TST by parents in tuberculosis contact tracing at 5 general hospitals. TST was performed with PPD RT 232 TU (AJ Vaccines, Denmark) and IGRA was used with the QuantiFeron TB Gold in tube (QFT) (Qiagen, Germany).

$\checkmark$ TSTs were considered positive if induration $\geq 10 \mathrm{~mm}$ on the first test or demonstrated an increase $\geq 6 \mathrm{~mm}$ over the induration of the $1^{\text {st }}$ TST on repeat testing after 8 weeks.

The agreement between TST and QFT was evaluated using proportion agreement and kappa statistic. The positive prevalence and agreement was subanalyzed according to different age groups and estimated by cross tabulation.

\section{RESULTS}

$\checkmark$ A total of 80 subjects were recruited and a total of 110 tests were conducted. Forty-two (52.5\%) were male, median age was 4 years of age. The percentage of intrafamilial and non-intrafamilial contact was $82.5 \%(66 / 80)$ and $17.5 \%(14 / 80)$.

The result for AFB smear of index cases was positive in $33.8 \%(27 / 80)$, negative in $53.8 \%$ $(43 / 80)$ and unknown in $12.5 \%(10 / 80)$. Lung cavity was seen in $27.5 \%(22 / 80)$ of index cases, $41.3 \%(33 / 80)$ had no cavity, and $31.2 \%(25 / 80)$ of the cases were unknown. $\checkmark$ In 80 subjects tested, the positive rate of TST was $43.8 \%$ (35/80), but the positive rate of QFT was only $5 \%$ (4/80). All 4 QFT positive subjects were positive for TST.

$\checkmark$ The overall agreement between TST and QFT was very low, showing positive or negative concordance rate in 75/110 tests (68.2\%) and the kappa agreement was 0.1309 (95\% Cl: 0.006 0.268) (Table 1). TST positive but QFT negative discordance (31/32, 96.8\%) occurred far more frequently than TST negative but QFT positive negative discordance $(1 / 32,3.1 \%)$.

$\checkmark$ The positive prevalence estimated by either TST or QFT showed no significant difference between children aged 0 to 4 years old and $\geq 5$ years old. The positive rate of QFT was much lower than that of TST in both groups (Table 2).
Table 1. Agreement between TST and QFT in 110 tests on 80 subjects enrolled in contact tracing

\begin{tabular}{|c|c|c|c|}
\hline & QFT (+) & QFT (-) & Total \\
\hline TST (+) & $4(3.6 \%)$ & $31(28.2 \%)$ & $35(31.8 \%)$ \\
\hline TST (-) & $1(1.0 \%)$ & $74(67.3 \%)$ & $75(68.2 \%)$ \\
\hline Total & $5(4.6 \%)$ & $105(95.4 \%)$ & $110(100 \%)$ \\
\hline
\end{tabular}

$\mathrm{K}=0.1309(95 \% \mathrm{Cl}-0.006$ to 0.268$)$
Table 2. Positive rate of TST and QFT in children aged $<5$ years and $\geq 5$ years old

\begin{tabular}{|r|c|c|c|}
\hline & $0-4$ yrs $(n=43)$ & $\geq 5$ yrs $(n=37)$ & $p$-value \\
\hline TST (+) & $20(46.5 \%)$ & $15(40.5 \%)$ & 0.756 \\
\hline$(-)$ & $23(53.5 \%)$ & $22(59.5 \%)$ & \\
\hline QFT (+) & $2(4.7 \%)$ & $2(5.4 \%)$ & 1.000 \\
\hline$(-)$ & $41(95.3 \%)$ & $35(94.6 \%)$ & \\
\hline
\end{tabular}

\section{CONCLUSIONS}

$\checkmark$ Latent TB infection rate estimated by either TST or QFT showed no significant difference regardless of age.

$\checkmark$ In both groups of age, QFT positivity was much lower than TST positivity in this study. It was concluded that the use of QTF alone in children, especially younger than 5 years of age in tuberculosis contact tracing could not still acceptable due to the low concordance between TST and QTF. 